\title{
Learned taste aversions induced by rotational stimulation*
}

\author{
J. JAY BRAUN and HARRIS McINTOSH JR. \\ Yale University, New Haven, Connecticut 06510
}

\begin{abstract}
Rats presented with a distinctive taste cue (salt or sucrose) followed by rotational stimulation subsequently displayed an aversion to the specific cue that had been paired with rotation. Evidence of such learning was noted after a single trial when the taste cue and rotational treatment had been presented in separate places several minutes apart. This observation was attested to through comparisons of four groups of rats, two of which received a specific and different taste cue paired with rotation, one which received rotational stimulation paired with plain water, and one which was not rotated. A discrimination procedure was employed in which the consumption of all solutions (salt, sucrose, and water) was measured for all rats throughout eight training and two testing cycles.
\end{abstract}

A variety of drugs and toxins and radiation treatment serve as highly effective USs in taste aversion learning (e.g., Garcia \& Koelling, 1967), and these treatments presumably have in common gastrointestinal or central consequences associated with sickness, consequences for which flavor stimuli appear to have a special associative affinity (Garcia \& Ervin, 1968; Seligman, 1970). Green and Rachlin (1973) recently have presented evidence suggesting that motion sickness induced by rotational stimulation belongs with this list of treatments.

Motion sickness qualitatively resembles other kinds of sickness (Speigel, 1946), and it can be induced readily in many animals (Tyler \& Bard, 1949). It appears to be produced via vestibular stimulation, since animals do not display motion sickness following labyrinthectomy (Sjoberg, 1929), nor is it displayed by deaf humans who lack vestibular sensitivity (McNally \& Stuart, 1942). Therefore, in contrast to other sickness-inducing treatments, the relevant peripheral receptors for motion sickness can be specified.

An additional feature of particular interest here is that motion stimulation is accompanied by many peripheral cues related to the treatment per se (visual, kinesthetic), and these cues should be readily apparent to a $S$ and contiguous with the onset of sickness. This contiguity could inform $\mathrm{S}$ that movement, rather than something consumed prior to treatment, is associated with (caused) the malaise, and this feature of motion stimulation contrasts with treatments like irradiation for which such treatment-related cues appear to be relatively subtle and ambiguous. However, if rats are especially "prepared" (Seligman, 1970) to respond to sickness as a consequence of prior consummatory behavior and attendant taste cues regardless of how the sickness is caused or how apparent its nonconsummatory cause (Garcia \& Ervin, 1968), then motion sickness should serve readily as a US in taste aversion learning even when presentation of taste cue and treatment do not overlap.

The present study used a discrimination procedure to assess the presence and specificity of taste aversions

*Supported by USPHS Grant NS 08658 to J.J.B. Request reprints from J. Braun, Department of Psychology. Arizona State University, Tempe, Arizona 85281. produced by paired rotational stimulation in rats. In order to diminish relationships of context and contiguity between ingestion cues and rotational treatment, these events were temporally and spatially separated by presenting a taste solution (the CS) in the rats' home cages prior to subjecting them to rotational treatment (the US) in a separate cage and room.

\section{METHOD}

Twenty-two male Long-Evans hooded rats, 17 of which were 93 days of age and the remainder of which were 150-180 days, were used as Ss. They were housed individually with free access to food throughout the experiment.

The apparatus for producing the rotational stimulation used as the US was a record turntable converted to revolve at $150 \mathrm{rpm}$. A cylindrical cage, $18 \mathrm{~cm} \times 24 \mathrm{~cm}$ in diam, with a wire mesh top was affixed to the turntable and divided into two compartments with a sheet metal barrier. In order to diminish rotational adaptation and to ensure changes in inertia relative to gravity throughout a stimulation period, the entire apparatus was displaced to an angle of 20 deg from horizontal.

Drinking tubes calibrated in milliliters and fitted with rubber stoppers and $4-\mathrm{cm}$ stainless steel drinking spouts were used for measurements of fluid consumption.

Aqueous solutions of $50 \mathrm{~g} /$ liter sucrose and $9 \mathrm{~g} /$ liter sodium chloride of A.C.S. grade at room temperature $\left(22^{\circ}-24^{\circ} \mathrm{C}\right)$ were used as stimuli. These two taste solutions were chosen because pilot studies indicated that they had approximately equivalent hedonic value for rats in one-bottle tests under conditions highly similar to those in the present experiment and because both taste solutions are preferred to water by male hooded rats. The third drinking solution was tap water.

\section{Procedure}

A discrimination procedure highly similar to that used in a previous study (Braun, Slick. \& Lorden, 1972) was employed here to test whether a taste stimulus paired with rotational stimulation would be avoided relative to a stimulus that was not so paired. All Ss were accustomed for 8 days to a water-deprivation schedule of $15 \mathrm{~min}$ of free drinking every $12 \mathrm{~h}$. They were then divided into four groups matched on the basis of age and mean water consumption on the last three drinking sessions. One-bottle drinking tests of 15 min duration were used, and all drinking occurred in the home cages throughout the experiment.

The training and testing regime consisted of eight cycles of presenting one trial each of sucrose, salt, and water, in that order, to every rat in the study, with a single solution presented 


\section{GROUPS SUR \& STR COMBINED}

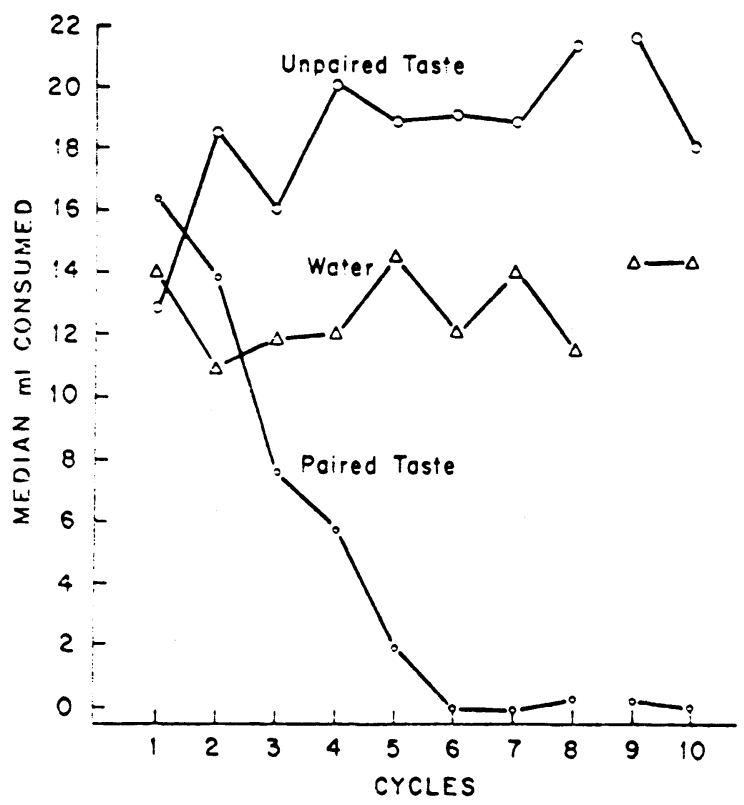

Fig. 1. Mean milliliters consumed per cycle of each of three drinking solutions by rats $(N=12)$ that received rotational stimulation following exposure to a particular taste cue (paired taste). but not following exposure to the other cue (unpaired taste). Each cycle represents three 15 -min trials. one per solution. The paired taste was always presented and measured prior to rotational stimulation, and one paired trial was given every $36 \mathrm{~h}$. On Cycles 9 and 10, trial order was changed and rotation was not presented.

every trial (every $12 \mathrm{~h}$ ). Each cycle therefore represents three trials: one trial per drinking solution.

Group SuR $(N=6)$ was subjected to rotational stimulation following each sucrose trial and remained in the home cage following salt and water trials. Similarly. Group StR $(N=6)$ was subjected to rotational stimulation following salt trials and remained in the home cage following sucrose and water trials. On rotation trials, the rats were removed from their home cages within 1-3 min following the termination of the 15-min drinking trial and placed in the rotation apparatus in a separate room for $5 \mathrm{~min}$ of stimulation. Since the rats typically stopped drinking and began eating within $9-12 \mathrm{~min}$ after the bottle had been presented. the temporal separation between offset of CS and onset of LS was greater than $3 \mathrm{~min}$. A control group. $C(N=6)$. was not rotated but was removed from the home cage and placed in a cage adjacent to the operating rotation apparatus for $5 \mathrm{~min}$ without food after one trial within every cycle. Finally; an additional group. WR. Was subjected to rotational stimulation following water drinking trials but not following salt or sucrose trials.

Because there was an odd number of trials within a cycle and because a trial was given every $12 \mathrm{~h}$, the training trials (every third trial) alternated between morning and evening presentations. hence reducing the possibility of circadian cues signaling the time of an impending trial. Nonetheless, since trial order was not systematically varied during the first eight cycles, two additional test cycles were run for all rats (Cycles 9 and 10) with two critical changes from the preceding eight cycles: (1) Rotational stimulation was discontinued, and this tested how well drinking suppression would be maintained when the LS was withdrawn; (2) trial order was differentially scrambled for each rat so that on Cycle 9 the CS would occur either two or four trials bey ond the last CS rather than three trials. as had been the case for the previous eight cycles. In addition. the CS was arranged to follow the test solution that it had not followed previously. This allowed testing of the degree to which suppression of drinking in the experimental groups was specific to the CS (salt or sucrose). as opposed to its being cued by. stimuli associated with the invariant order of presentation of the previous eight cycles.

An additional group of four naive male Long-Evans rats. Group TN. was run concurrently with, but separate from, the main study as a preliminary test of whether traumatic stimulation other than rotation. $5 \mathrm{~min}$ of loud noise. would produce aversions to tastes using the training paradigm described above. Two Ss received noise paired with the sucrose stimulus and two Ss received noise paired with the salt. The noise stimulus was produced by an electric horn within an enclosed double-chambered sound-attenuated box $(70 \times 75 \times 70 \mathrm{~cm})$ in a room separate from the one in which drinking measures were taken. The horn produced a broad frequency range at $105 \mathrm{~dB}$ peak output.

Statistical treatment of the data was accomplished using the Mann-Whitney $\mathrm{L}$ test for comparisons between groups, and the Wilcoxon $T$ test was used for comparisons within groups. Reported probability values are two-tailed (Siegel, 1956).

\section{RESULTS}

Rotational treatment produced clear signs of disorganization independent of drinking behavior. These included an inability of rotated Ss to assume a normal posture for about $1 \mathrm{~min}$ following treatment and a lack of eating during a 15 -min observation period when the rats were returned to their home cages. During this period, they tended to huddle against the floor in the rear of the cage, whereas control animals removed from their home cages for $5 \mathrm{~min}$ but not rotated tended to eat immediately upon being returned. Huddling and quiescence were noted also following noise treatment in Group TN. Rotated rats (Groups StR and SuR combined) showed also a lower percentage weight gain (median $=3.1 \%$ ) than did the untreated control rats (median $=11.2 \%)$ over a 12-day period spanning training and testing $(\mathrm{U}=13 . \mathrm{p}<.05)$.

The critical comparison for an assessment of the effectiveness of rotational stimulation as a US was amount consumed of the stimulus that was paired with rotation vs amount consumed of the unpaired stimulus in Groups SuR and StR. This comparison is portrayed in Fig. 1, in which the performances of the two groups are combined: it is noted that significantly less of the paired taste was consumed after only one pairing trial (Cycle 2. $\mathrm{T}=5.5, p<.01)$. This difference became more pronounced with subsequent trials and persisted when trial order was scrambled on Cycles 9 and $10(\mathrm{~T}=0$. $\mathrm{p}<.01)$. In addition, by Cycle 3 it was apparent that Groups SuR and StR drank less of the paired taste stimulus than of the water $(T=0, p<.01)$. whereas the opposite was the case for the unpaired taste cue which was preferred to water $(T=1 . p<.01)$. The Cycle 1 difference between paired and unpaired taste cues (see Fig. 1) was a nonsignificant chance event $(T=25)$.

Group $C$, which was not subjected to rotational stimulation. preferred both taste stimuli to plain water 
as measured by mean amount consumed of each taste solution per rat per trial (sucrose, $19.9 \mathrm{ml}$; salt, $17.4 \mathrm{ml}$ ), compared to water consumption $(12.9 \mathrm{ml})$ over the 10 cycles $(\mathrm{N}=6, \mathrm{~T}=0, \mathrm{p}<.05$, both stimuli), and this group consumed approximately equal amounts of each (see also Table 1).

Table 1 presents the average Cycle 9 consumption of each solution by each group in the main experiment. Recall that on this cycle. order of presentation of the three solutions had been changed. Note that Groups SuR and $S t R$ consumed markedly less of the respective taste solutions that were paired with rotation compared to the other groups $(\mathrm{U}=0, \mathrm{p}=.002$ for both). Consumption of nonpaired tastes was highly similar across groups. Surprisingly, water appeared to develop cueing properties for Group.WR which drank less water than any other group. This difference was not as marked as the results with the taste solutions, but it approached significance $(\mathrm{U}=3.5, \mathrm{p}<.10$ compared to Group $\mathrm{C})$, and because of a consistently decreasing trend in the water drinking of this group from Day 1 (median = $13.5 \mathrm{ml})$ to Day $9(7 \mathrm{ml})$, it is believed that a clearly significant difference would have developed with continued training.

The four Ss run separately to test the effects of pairing a particular taste cue with loud noise, a presumably noxious event which was not deemed likely to produce sickness, displayed no indication of associating a taste cue with noise treatment: No trend was observed for the paired taste across cycles, and Cycle 9 consumption of the paired taste was essentially equal to consumption of the unpaired taste (paired taste, median $=17 \mathrm{ml}$; unpaired taste, median $=16 \mathrm{ml}$; water, median $=11 \mathrm{ml}$ ).

\section{DISCUSSION}

The significant and increasing tendency of the experimental rats to avoid the taste solution that had occurred prior to rotational stimulation clearly indicates that such stimulation is an effective US for taste aversion learning, thus supporting the observations of Green and Rachlin (1973). The efficacy of the procedure is attested to by the development of a significant aversion to the paired taste after only one trial and by the persistence of the aversion when trial order was scrambled on Cycles 9 and 10. In addition, taste aversions produced in this manner were specific to the paired taste cue and did not generalize to the unpaired and normally equally palatable taste cue.

Motion-sick humans tend to ascribe their malaise to such things as "odors, overheated compartments, visceral sensations, disagreeable sights, [or] distasteful food [Tyler \& Bard, 1949, p. 317] " which may have occurred prior to or during motion stimulation. The present data are in accord with the spirit of the above observations: The many cues contiguous with rotational stimulation do not appear to block the salience of a prior taste as a
Table 1

Median/Mean Milliliters of Each Solution Consumed on Cycle 9 Rounded to Nearest Milliliter for Each Group*

\begin{tabular}{llccc}
\hline Group & N & Sucrose & Salt & Water \\
\hline C & 6 & $24 / 23$ & $21 / 19$ & $13 / 12$ \\
SuR & 6 & $1 / 5$ & $22 / 23$ & $15 / 13$ \\
StR & 6 & $19 / 20$ & $0 / 2$ & $12 / 13$ \\
WR & 4 & $22 / 21$ & $19 / 19$ & $7 / 5$ \\
\hline
\end{tabular}

${ }^{*} C=$ control, SuR $=$ sucrose paired with rotation, $S t R=$ salt paired with rotation, $W R=$ water paired with rotation.

CS for such stimulation. In addition, it is clear that the noxious stimulation need not be directly relevant to, overlap with, or occur in the same environment as ingestion in order to be an effective US when paired with flavor cues.

Since rats do not vomit (e.g., Garcia \& Ervin, 1968), independent criteria for the effectiveness of motion stimulation (or any other treatment) for producing nausea are not as obvious as with animals that do. Therefore, the suggestion that repetitive motion stimulation produces sickness cues in rats is an inference from studies conducted on animals that do vomit. Although other symptoms-huddling, quiescence, and lack of eating-were similar to those observed following toxic drug and irradiation treatments, it is recognized that such indices need not obviously be present in order for effective conditioning to occur (Berger, 1972; Braun $\&$ Snyder, 1973) and that the presence of such symptoms does not insure successful taste aversion learning (Group TN in the present study). Therefore, the validity of these general indices is questionable.

However, the possibility that the overall level of trauma produced by rotation, rather than any nausea-related effects, provided the effective US is indirectly diminished by the following observations: (1) the total lack of effectiveness of 5 min of "traumatic noise" stimulation in Group TN; (2) Garcia and Koelling's (1966) observation that electric shock was an ineffective US for taste when consumption of the taste solution paired with shock was tested, after training, without shock present; (3) the effectiveness of rotation in the present study after only a single pairing trial when administered in a totally separate setting from, and minutes after, exposure to the taste cue; and (4) the fact that repetitive motion stimulation similar to that presented here induces emesis in animals other than rats (Tyler \& Bard, 1949).

As a highly effective US for taste aversion learning, motion stimulation has two important features in common with a majority of other effective treatments: First, as noted above, it induces emesis in animals that can vomit, and second, emesis and other signs of visceral discomfort provoked by such treatments are eliminated by small lesions of the "chemoreceptive trigger zone" (Borison \& Wang, 1953) of the area postrema in the medulla oblongata. Such widely diverse treatments as apomorphine injection (Wang \& Borison, 1952). 
irradiation (Chinn \& Wang, 1954), and repetitive swinging motion (Wang \& Chinn. 1954), all of which readily induce emesis in normal dogs or cats, are ineffective following such lesions. A recent study by Berger. Wise, and Stein (1973) has indicated that the area postrema of the rat may be necessary for the development of taste aversions following treatment with some drugs. Lesions of this area probably also will be found to block taste aversion learning in rats for all treatments which have emetic consequences in other animals and which also serve as highly effective USs in taste aversion learning. Future observations supporting this suggestion would serve to relate the taste aversion learning phenomenon (Garcia \& Ervin. 1968) to the general literature on mechanisms of nausea and thereby contribute to understanding the unconditioned basis of such aversions.

\section{REFERENCES}

Berger, G. Conditioning of food aversions by injections of psychoactive drugs. Journal of Comparative \& Physiological Psychology. 1972, 81, 21-26.

Berger, B. D., Wise, C. D., \& Stein, L. Area postrema damage and bait shyness. Journal of Comparative \& Physiological Psychology, 1973, 82, 475-479.

Borison. H. L., \& Wang, S. C. Physiology and pharmacology of romiting. Pharmacological Reviews, 1953, 5, 193-230.

Braun. J. J., Slick, T. B., \& Lorden, J. F. Involvement of gustatory neocortex in the learning of taste aversions. Phrsiology \& Behavior. 1972, 9.637-641.

Braur, J. J., \& Snyder, D. R. Taste aversions and acute methyl mercury poisoning in rats. Bulletin of the Psychonomic Society, 1973, 1. 419-420.

Chinn. H. I., \& Wang. S. C. Locus of emetic action following irradiation. Proceedings of the Society of Experimental Biology \& Medicine. 1954. 85, 472-474.

Garcia. J.. \& Ervin. F. Gustatory-visceral and telereceptor-cutaneous conditioning-Adaptation in internal and external mileus. Communications in Behavioral Biology. 1968. 1. 389-415.

Garcia. J.. \& Koelling. R. A. Relation of cue to consequence in avoidance learning. Psychonomic Science, 1966. 4. 123-124.

Garcia, J., \& Koelling. R. A. A comparison of aversions induced by $\mathrm{X}$ rays. toxins, and drugs in the rat. Radiation Research Supplement. 1967, 7. 439-450.

Green, L.. \& Rachlin, H. The effect of rotation on the learning of taste aversions. Bulletin of the Psychonomic Society. 1973. 1. $137-138$.

McNally. W. J., \& Stuart. E. A. Physiology of the labyrinth reviewed in relation to sea sickness and other forms of motion sickness. War Medicine, 1942, 2, 683-771.

Seligman, M. E. P. On the generality of the laws of learning. Psychological Review, 1970. 77. 406-418.

Siegel. S. Nonparametric statistics for the behavioral sciences. New York: McGraw-Hill, 1956.

Sjoberg, A. A. Experimental studies of the eliciting mechanism of sea sickness. Acta Oto-Laryngologica. 1929, 13, 343-347.

Speigel, E. A. Effect of labyrinthine reflexes on the vegetative nervous system. Archives of Otolaryngology, 1946, 44. 61-72.

Tyler, D. B., \& Bard, P. Motion sickness. Physiological Reviews. 1949. 29, 311-369.

Wang, S. C., \& Borison. H. L. A new concept of organization of the central emetic mechanism: Recent studies on the sites of action of apomorphine. copper sulfate \& cardiac glycosides. Gastroenterology, 1952, 22, 1-12.

Wang, S. C.. \& Chinn, H. I. Experimental motion sickness in dogs: Functional importance of chemoreceptive emetic trigger zone. American Journal of Physiology. 1954. 178. 111-116.

(Received for publication April 26. 1973; accepted May 10. 1973.) 